\title{
Pascal's Theorem in Real Projective Plane
}

\author{
Roland Coghetto \\ Rue de la Brasserie 5 \\ 7100 La Louvière, Belgium
}

\begin{abstract}
Summary. In this article we check, with the Mizar system 2, Pascal's theorem in the real projective plane (in projective geometry Pascal's theorem is also known as the Hexagrammum Mysticum Theorem ${ }^{1}$ Pappus' theorem is a special case of a degenerate conic of two lines.

For proving Pascal's theorem, we use the techniques developed in the section "Projective Proofs of Pappus' Theorem" in the chapter "Pappus' Theorem: Nine proofs and three variations" 11. We also follow some ideas from Harrison's work. With HOL Light, he has the proof of Pascal's theorem ${ }^{2}$. For a lemma, we use PROVERS ${ }^{3}$ and OTT2MIZ by Josef Urbar ${ }^{4}[12,6$, 7]. We note, that we don't use Skolem/Herbrand functions (see "Skolemization" in [1]).
\end{abstract}

MSC: 51E15 51N15 03B35

Keywords: Pascal's theorem; real projective plane; Grassman-Plücker relation MML identifier: PASCAL, version: 8.1.06 5.43.1297

\section{Preliminaries}

From now on $n$ denotes a natural number, $K$ denotes a field, $a, b, c, d, e, f$, $g, h, i, a_{1}, b_{1}, c_{1}, d_{1}, e_{1}, f_{1}, g_{1}, h_{1}, i_{1}$ denote elements of $K, M, N$ denote square matrices over $K$ of dimension 3 , and $p$ denotes a finite sequence of elements of $\mathbb{R}$.

Now we state the propositions:

(1) Let us consider points $p, q, r$ of $\mathcal{E}_{\mathrm{T}}^{3}$. Then

\footnotetext{
1 https://en.wikipedia.org/wiki/Pascal's_theorem

2 https://github.com/jrh13/hol-light/tree/master/100/pascal.ml

3 https://www.cs.unm.edu/ ${ }^{2}$ ccune/prover9/

${ }^{4}$ https://github.com/JUrban/ott2miz 
(i) $\langle|p, q, r|\rangle=\langle|r, p, q|\rangle$, and

(ii) $\langle|p, q, r|\rangle=\langle|q, r, p|\rangle$.

(2) Suppose $\langle\langle a, b, c\rangle,\langle d, e, f\rangle,\langle g, h, i\rangle\rangle=\left\langle\left\langle a_{1}, b_{1}, c_{1}\right\rangle,\left\langle d_{1}, e_{1}, f_{1}\right\rangle,\left\langle g_{1}, h_{1}, i_{1}\right\rangle\right\rangle$. Then

(i) $a=a_{1}$, and

(ii) $b=b_{1}$, and

(iii) $c=c_{1}$, and

(iv) $d=d_{1}$, and

(v) $e=e_{1}$, and

(vi) $f=f_{1}$, and

(vii) $g=g_{1}$, and

(viii) $h=h_{1}$, and

(ix) $i=i_{1}$.

(3) There exists $a$ and there exists $b$ and there exists $c$ and there exists $d$ and there exists $e$ and there exists $f$ and there exists $g$ and there exists $h$ and there exists $i$ such that $M=\langle\langle a, b, c\rangle,\langle d, e, f\rangle,\langle g, h, i\rangle\rangle$.

(4) Suppose $M=\langle\langle a, b, c\rangle,\langle d, e, f\rangle,\langle g, h, i\rangle\rangle$. Then

(i) $a=M_{1,1}$, and

(ii) $b=M_{1,2}$, and

(iii) $c=M_{1,3}$, and

(iv) $d=M_{2,1}$, and

(v) $e=M_{2,2}$, and

(vi) $f=M_{2,3}$, and

(vii) $g=M_{3,1}$, and

(viii) $h=M_{3,2}$, and

(ix) $i=M_{3,3}$.

(5) Suppose $M=\langle\langle a, b, c\rangle,\langle d, e, f\rangle,\langle g, h, i\rangle\rangle$. Then $M^{\mathrm{T}}=\langle\langle a, d, g\rangle,\langle b, e, h\rangle$, $\langle c, f, i\rangle\rangle$. The theorem is a consequence of (4) and (3).

(6) Suppose $M=\langle\langle a, b, c\rangle,\langle d, e, f\rangle,\langle g, h, i\rangle\rangle$ and $M$ is symmetric. Then

(i) $b=d$, and

(ii) $c=g$, and

(iii) $h=f$.

The theorem is a consequence of (5) and (2). 
(7) Let us consider square matrices $M, N$ over $\mathbb{R}_{\mathrm{F}}$ of dimension 3 . If $N$ is symmetric, then $M^{\mathrm{T}} \cdot N \cdot M$ is symmetric.

(8) Let us consider a square matrix $M$ over $\mathbb{R}_{\mathrm{F}}$ of dimension 3 , elements $a$, $b, c, d, e, f, g, h, i, x, y, z$ of $\mathbb{R}_{\mathrm{F}}$, an element $v$ of $\mathcal{E}_{\mathrm{T}}^{3}$, a finite sequence $u_{10}$ of elements of $\mathbb{R}_{\mathrm{F}}$, and a finite sequence $p$ of elements of $\mathbb{R}^{1}$. Suppose $p=M \cdot u_{10}$ and $v=\operatorname{M} 2 \mathrm{~F}(p)$ and $M=\langle\langle a, b, c\rangle,\langle d, e, f\rangle,\langle g, h, i\rangle\rangle$ and $u_{10}=\langle x, y, z\rangle$. Then

(i) $p=\langle\langle a \cdot x+(b \cdot y)+(c \cdot z)\rangle,\langle d \cdot x+(e \cdot y)+(f \cdot z)\rangle,\langle g \cdot x+(h \cdot y)+(i \cdot z)\rangle\rangle$, and

(ii) $v=\langle a \cdot x+(b \cdot y)+(c \cdot z), d \cdot x+(e \cdot y)+(f \cdot z), g \cdot x+(h \cdot y)+(i \cdot z)\rangle$.

(9) Let us consider a square matrix $M$ over $\mathbb{R}$ of dimension 3 , and elements $a, b, c, d, e, f, g, h, i, p_{1}, p_{2}, p_{3}$ of $\mathbb{R}$. Suppose $M=\langle\langle a, b, c\rangle,\langle d, e, f\rangle$, $\langle g, h, i\rangle\rangle$ and $p=\left\langle p_{1}, p_{2}, p_{3}\right\rangle$. Then $M \cdot p=\left\langle a \cdot p_{1}+\left(b \cdot p_{2}\right)+\left(c \cdot p_{3}\right)\right.$, $\left.d \cdot p_{1}+\left(e \cdot p_{2}\right)+\left(f \cdot p_{3}\right), g \cdot p_{1}+\left(h \cdot p_{2}\right)+\left(i \cdot p_{3}\right)\right\rangle$.

\section{Conic in Real Projective Plane}

Let $a, b, c, d, e, f$ be real numbers and $u$ be an element of $\mathcal{E}_{\mathrm{T}}^{3}$. The functor qfconic $(a, b, c, d, e, f, u)$ yielding a real number is defined by the term

(Def. 1) $a \cdot u(1) \cdot u(1)+(b \cdot u(2) \cdot u(2))+(c \cdot u(3) \cdot u(3))+(d \cdot u(1) \cdot u(2))+(e \cdot$ $u(1) \cdot u(3))+(f \cdot u(2) \cdot u(3))$.

The functor conic $(a, b, c, d, e, f)$ yielding a subset of the projective space over $\mathcal{E}_{\mathrm{T}}^{3}$ is defined by the term

(Def. 2) $\left\{P\right.$, where $P$ is a point of the projective space over $\mathcal{E}_{\mathrm{T}}^{3}$ : for every element $u$ of $\mathcal{E}_{\mathrm{T}}^{3}$ such that $u$ is not zero and $P=$ the direction of $u$ holds qfconic $(a, b, c, d, e, f, u)=0\}$.

In the sequel $a, b, c, d, e, f$ denote real numbers, $u, u_{1}, u_{2}$ denote non zero elements of $\mathcal{E}_{\mathrm{T}}^{3}$, and $P$ denotes an element of the projective space over $\mathcal{E}_{\mathrm{T}}^{3}$.

Now we state the propositions:

(10) Suppose the direction of $u_{1}=$ the direction of $u_{2}$ and qfconic $\left(a, b, c, d, e, f, u_{1}\right)=0$. Then $\operatorname{qfconic}\left(a, b, c, d, e, f, u_{2}\right)=0$.

(11) If $P=$ the direction of $u$ and $\operatorname{qfconic}(a, b, c, d, e, f, u)=0$, then $P \in$ $\operatorname{conic}(a, b, c, d, e, f)$. The theorem is a consequence of (10).

Let $a, b, c, d, e, f$ be real numbers. The functor symmetric $3(a, b, c, d, e, f)$ yielding a square matrix over $\mathbb{R}_{\mathrm{F}}$ of dimension 3 is defined by the term

(Def. 3) $\langle\langle a, d, e\rangle,\langle d, b, f\rangle,\langle e, f, c\rangle\rangle$.

Now we state the propositions: 
(12) symmetric3 $(a, b, c, d, e, f)$ is symmetric. The theorem is a consequence of (5).

(13) Let us consider real numbers $a, b, c, d, e, f$, a point $u$ of $\mathcal{E}_{\mathrm{T}}^{3}$, and a square matrix $M$ over $\mathbb{R}$ of dimension 3. Suppose $p=u$ and $M=$ symmetric $3(a, b, c, d, e, f)$.

Then SumAll QuadraticForm $(p, M, p)=\operatorname{qfconic}(a, b, c, 2 \cdot d, 2 \cdot e, 2 \cdot f, u)$.

(14) Let us consider an invertible square matrix $N$ over $\mathbb{R}_{\mathrm{F}}$ of dimension 3 , square matrices $N_{1}, M_{1}, M_{2}$ over $\mathbb{R}$ of dimension 3 , and real numbers $a, b$, $c, d, e, f$. Suppose $N_{1}=\left(\mathbb{R}_{\mathrm{F}} \rightarrow \mathbb{R}\right) N$ and $M_{1}=\operatorname{symmetric} 3\left(a, b, c, \frac{d}{2}, \frac{f}{2}, \frac{e}{2}\right)$ and $M_{2}=\left(\mathbb{R}_{\mathrm{F}} \rightarrow \mathbb{R}\right)\left(\left(\mathbb{R} \rightarrow \mathbb{R}_{\mathrm{F}}\right) N_{1}^{\mathrm{T}}\right)^{\smile} \cdot M_{1} \cdot\left(\mathbb{R}_{\mathrm{F}} \rightarrow \mathbb{R}\right)\left(\left(\mathbb{R} \rightarrow \mathbb{R}_{\mathrm{F}}\right) N_{1}\right)^{\smile}$. Then $\left(\mathbb{R} \rightarrow \mathbb{R}_{\mathrm{F}}\right) M_{2}$ is symmetric.

Proof: $\left(\left(\mathbb{R} \rightarrow \mathbb{R}_{\mathrm{F}}\right) N_{1}^{\mathrm{T}}\right)^{\mathrm{T}}=\left(\mathbb{R} \rightarrow \mathbb{R}_{\mathrm{F}}\right) N_{1}$ by $\left[\underline{3}\right.$, (16)]. $\left(\mathbb{R} \rightarrow \mathbb{R}_{\mathrm{F}}\right) M_{2}$ is symmetric by [3, (16)], (12), (7).

(15) Let us consider real numbers $a_{1}, a_{2}, a_{3}, a_{4}, a_{5}, a_{6}, b_{1}, b_{2}, b_{3}, b_{4}, b_{5}, b_{6}$. Suppose symmetric $3\left(a_{1}, a_{2}, a_{3}, a_{4}, a_{5}, a_{6}\right)=\operatorname{symmetric} 3\left(b_{1}, b_{2}, b_{3}, b_{4}, b_{5}, b_{6}\right)$. Then

(i) $a_{1}=b_{1}$, and

(ii) $a_{2}=b_{2}$, and

(iii) $a_{3}=b_{3}$, and

(iv) $a_{4}=b_{4}$, and

(v) $a_{5}=b_{5}$, and

(vi) $a_{6}=b_{6}$.

The theorem is a consequence of (2).

(16) Let us consider real numbers $a, b, c, d, e, f$, a point $P$ of the projective space over $\mathcal{E}_{\mathrm{T}}^{3}$, and an invertible square matrix $N$ over $\mathbb{R}_{\mathrm{F}}$ of dimension 3. Suppose it is not true that $a=0$ and $b=0$ and $c=0$ and $d=0$ and $e=0$ and $f=0$. Suppose that $P \in \operatorname{conic}(a, b, c, d, e, f)$. Let us consider real numbers $f_{5}, f_{12}, f_{19}, f_{20}, f_{21}, f_{23}, f_{22}$, square matrices $M_{1}$, $M_{2}$ over $\mathbb{R}$ of dimension 3 , and a square matrix $N_{1}$ over $\mathbb{R}$ of dimension 3. Suppose $M_{1}=\operatorname{symmetric} 3\left(a, b, c, \frac{d}{2}, \frac{e}{2}, \frac{f}{2}\right)$ and $N_{1}=\left(\mathbb{R}_{\mathrm{F}} \rightarrow \mathbb{R}\right) N$ and $M_{2}=\left(\mathbb{R}_{\mathrm{F}} \rightarrow \mathbb{R}\right)\left(\left(\mathbb{R} \rightarrow \mathbb{R}_{\mathrm{F}}\right) N_{1}^{\mathrm{T}}\right)^{\smile} \cdot M_{1} \cdot\left(\mathbb{R}_{\mathrm{F}} \rightarrow \mathbb{R}\right)\left(\left(\mathbb{R} \rightarrow \mathbb{R}_{\mathrm{F}}\right) N_{1}\right)^{\smile}$ and $M_{2}=\operatorname{symmetric} 3\left(f_{5}, f_{21}, f_{23}, f_{12}, f_{19}, f_{22}\right)$. Then

(i) it is not true that $f_{5}=0$ and $f_{21}=0$ and $f_{23}=0$ and $f_{12}=0$ and $f_{22}=0$ and $f_{19}=0$, and

(ii) (the homography of $N)(P) \in \operatorname{conic}\left(f_{5}, f_{21}, f_{23}, 2 \cdot f_{12}, 2 \cdot f_{19}, 2 \cdot f_{22}\right)$.

Proof: Consider $Q$ being a point of the projective space over $\mathcal{E}_{\mathrm{T}}^{3}$ such that $P=Q$ and for every element $u$ of $\mathcal{E}_{\mathrm{T}}^{3}$ such that $u$ is not zero 
and $Q=$ the direction of $u$ holds $\operatorname{qfconic}(a, b, c, d, e, f, u)=0$. Reconsider $M=\operatorname{symmetric} 3\left(a, b, c, \frac{d}{2}, \frac{e}{2}, \frac{f}{2}\right)$ as a square matrix over $\mathbb{R}$ of dimension 3. Consider $u_{19}, v_{3}$ being elements of $\mathcal{E}_{\mathrm{T}}^{3}, u_{17}$ being a finite sequence of elements of $\mathbb{R}_{F}, p_{11}$ being a finite sequence of elements of $\mathbb{R}^{1}$ such that $P=$ the direction of $u_{19}$ and $u_{19}$ is not zero and $u_{19}=u_{17}$ and $p_{11}=N \cdot u_{17}$ and $v_{3}=\operatorname{M} 2 \mathrm{~F}\left(p_{11}\right)$ and $v_{3}$ is not zero and (the homography of $\left.N\right)(P)=$ the direction of $v_{3}$. Reconsider $p_{10}=u_{19}$ as a finite sequence of elements of $\mathbb{R} . \operatorname{SumAll}$ QuadraticForm $\left(p_{10}, M, p_{10}\right)=\operatorname{qfconic}\left(a, b, c, 2 \cdot \frac{d}{2}, 2 \cdot \frac{e}{2}, 2 \cdot \frac{f}{2}, u_{19}\right)$. Consider $a_{8}, b_{8}, c_{11}, d_{4}, e_{5}, f_{24}, g_{2}, h_{2}, i_{2}$ being elements of $\mathbb{R}_{\mathrm{F}}$ such that $N=\left\langle\left\langle a_{8}, b_{8}, c_{11}\right\rangle,\left\langle d_{4}, e_{5}, f_{24}\right\rangle,\left\langle g_{2}, h_{2}, i_{2}\right\rangle\right\rangle$. Reconsider $u_{10}=u_{17}$ as a finite sequence of elements of $\mathbb{R}$. Reconsider $N_{1}=\left(\mathbb{R}_{\mathrm{F}} \rightarrow \mathbb{R}\right) N$ as a square matrix over $\mathbb{R}$ of dimension 3 . Reconsider $M_{2}=\left(\mathbb{R}_{\mathrm{F}} \rightarrow \mathbb{R}\right)((\mathbb{R} \rightarrow$ $\left.\left.\mathbb{R}_{\mathrm{F}}\right) N_{1}^{\mathrm{T}}\right)^{\smile} \cdot M \cdot\left(\mathbb{R}_{\mathrm{F}} \rightarrow \mathbb{R}\right)\left(\left(\mathbb{R} \rightarrow \mathbb{R}_{\mathrm{F}}\right) N_{1}\right)^{\smile}$ as a square matrix over $\mathbb{R}$ of dimension 3. $\left(\left(\mathbb{R} \rightarrow \mathbb{R}_{\mathrm{F}}\right) N_{1}^{\mathrm{T}}\right)^{\mathrm{T}}=\left(\mathbb{R} \rightarrow \mathbb{R}_{\mathrm{F}}\right) N_{1}$ by $\left[3\right.$, (16)]. $\left(\mathbb{R} \rightarrow \mathbb{R}_{\mathrm{F}}\right) M_{2}$ is symmetric by [3, (16)], (12), (7). Consider $m_{1}, m_{2}, m_{3}, m_{4}, m_{5}, m_{6}$, $m_{7}, m_{8}, m_{9}$ being elements of $\mathbb{R}_{\mathrm{F}}$ such that $M_{2}=\left\langle\left\langle m_{1}, m_{2}, m_{3}\right\rangle,\left\langle m_{4}\right.\right.$, $\left.\left.m_{5}, m_{6}\right\rangle,\left\langle m_{7}, m_{8}, m_{9}\right\rangle\right\rangle \cdot m_{2}=m_{4}$ and $m_{3}=m_{7}$ and $m_{8}=m_{6}$. Reconsider $u_{3}=N_{1} \cdot u_{10}$ as an element of $\mathcal{E}_{\mathrm{T}}^{3} \cdot u_{3}$ is not zero by [5, (24)], [14, (59), (86)]. Reconsider $u_{2}=N_{1} \cdot u_{10}$ as a non zero element of $\mathcal{E}_{\mathrm{T}}^{3}$. Reconsider $f_{5}=m_{1}, f_{12}=m_{2}, f_{19}=m_{3}, f_{21}=m_{5}, f_{22}=m_{6}, f_{23}=m_{9}$ as a real number. qfconic $\left(f_{5}, f_{21}, f_{23}, 2 \cdot f_{12}, 2 \cdot f_{19}, 2 \cdot f_{22}, u_{2}\right)=0$. It is not true that $f_{5}=0$ and $f_{21}=0$ and $f_{23}=0$ and $2 \cdot f_{12}=0$ and $2 \cdot f_{22}=0$ and $2 \cdot f_{19}=0 \cdot u_{2}=v_{3}$. For every real numbers $u_{11}$, $u_{12}, u_{13}, u_{14}, u_{15}, u_{18}, u_{16}$ and for every square matrices $U_{1}, U_{2}$ over $\mathbb{R}$ of dimension 3 and for every square matrix $U_{3}$ over $\mathbb{R}$ of dimension 3 such that $U_{1}=\operatorname{symmetric} 3\left(a, b, c, \frac{d}{2}, \frac{e}{2}, \frac{f}{2}\right)$ and $U_{3}=\left(\mathbb{R}_{\mathrm{F}} \rightarrow \mathbb{R}\right) N$ and $U_{2}=\left(\mathbb{R}_{\mathrm{F}} \rightarrow \mathbb{R}\right)\left(\left(\mathbb{R} \rightarrow \mathbb{R}_{\mathrm{F}}\right) U_{3}^{\mathrm{T}}\right)^{\smile} \cdot U_{1} \cdot\left(\mathbb{R}_{\mathrm{F}} \rightarrow \mathbb{R}\right)\left(\left(\mathbb{R} \rightarrow \mathbb{R}_{\mathrm{F}}\right) U_{3}\right)^{\smile}$ and $U_{2}=$ symmetric $3\left(u_{11}, u_{15}, u_{18}, u_{12}, u_{13}, u_{16}\right)$ holds it is not true that $u_{11}=0$ and $u_{15}=0$ and $u_{18}=0$ and $u_{12}=0$ and $u_{16}=0$ and $u_{13}=0$. (the homography of $N)(P) \in \operatorname{conic}\left(u_{11}, u_{15}, u_{18}, 2 \cdot u_{12}, 2 \cdot u_{13}, 2 \cdot u_{16}\right)$.

(17) Let us consider real numbers $a, b, c, d, e, f$, points $P_{1}, P_{2}, P_{3}, P_{4}, P_{5}$, $P_{6}$ of the projective space over $\mathcal{E}_{\mathrm{T}}^{3}$, and an invertible square matrix $N$ over $\mathbb{R}_{\mathrm{F}}$ of dimension 3. Suppose it is not true that $a=0$ and $b=0$ and $c=0$ and $d=0$ and $e=0$ and $f=0$. Suppose that $P_{1}, P_{2}, P_{3}, P_{4}, P_{5}$, $P_{6} \in \operatorname{conic}(a, b, c, d, e, f)$. Then there exist real numbers $a_{2}, b_{2}, c_{2}, d_{2}, e_{2}$, $f_{2}$ such that

(i) it is not true that $a_{2}=0$ and $b_{2}=0$ and $c_{2}=0$ and $d_{2}=0$ and $e_{2}=0$ and $f_{2}=0$, and

(ii) (the homography of $N)\left(P_{1}\right)$, (the homography of $\left.N\right)\left(P_{2}\right)$, 
(the homography of $N)\left(P_{3}\right)$, (the homography of $\left.N\right)\left(P_{4}\right)$, (the homography of $N)\left(P_{5}\right)$, (the homography of $\left.N\right)\left(P_{6}\right) \in$ $\operatorname{conic}\left(a_{2}, b_{2}, c_{2}, d_{2}, e_{2}, f_{2}\right)$.

The theorem is a consequence of (3), (14), (6), and (16).

From now on $a, b, c, d, e, f, g, h, i$ denote elements of $\mathbb{R}_{\mathrm{F}}$.

Now we state the proposition:

(i) if qfconic $(a, b, c, d, e, f,[1,0,0])=0$, then $a=0$, and

(ii) if qfconic $(a, b, c, d, e, f,[0,1,0])=0$, then $b=0$, and

(iii) if $\operatorname{qfconic}(a, b, c, d, e, f,[0,0,1])=0$, then $c=0$, and

(iv) if qfconic $(0,0,0, d, e, f,[1,1,1])=0$, then $d+e+f=0$.

\section{PASCAL's THEOREM}

In the sequel $M$ denotes a square matrix over $\mathbb{R}_{\mathrm{F}}$ of dimension $3, e_{1}, e_{2}, e_{3}$, $f_{1}, f_{2}, f_{3}$ denote elements of $\mathbb{R}_{\mathrm{F}}, M_{8}, M_{14}, M_{20}, M_{21}, M_{22}, M_{19}, M_{13}, M_{10}, M_{9}$, $M_{12}, M_{16}, M_{17}, M_{11}, M_{15}, M_{18}$ denote square matrices over $\mathbb{R}_{\mathrm{F}}$ of dimension 3, and $r_{1}, r_{2}$ denote real numbers.

Now we state the proposition:

(19) Suppose $M_{9}=\left\langle\langle 1,0,0\rangle,\langle 0,1,0\rangle,\left\langle e_{1}, e_{2}, e_{3}\right\rangle\right\rangle$ and $M_{12}=\langle\langle 1,0,0\rangle,\langle 0,0$, $\left.1\rangle,\left\langle f_{1}, f_{2}, f_{3}\right\rangle\right\rangle$ and $M_{16}=\left\langle\langle 0,1,0\rangle,\langle 1,1,1\rangle,\left\langle f_{1}, f_{2}, f_{3}\right\rangle\right\rangle$ and $M_{17}=\langle\langle 0,0$, $\left.1\rangle,\langle 1,1,1\rangle,\left\langle e_{1}, e_{2}, e_{3}\right\rangle\right\rangle$ and $M_{10}=\left\langle\langle 1,0,0\rangle,\langle 0,1,0\rangle,\left\langle f_{1}, f_{2}, f_{3}\right\rangle\right\rangle$ and $M_{11}=$ $\left\langle\langle 1,0,0\rangle,\langle 0,0,1\rangle,\left\langle e_{1}, e_{2}, e_{3}\right\rangle\right\rangle$ and $M_{15}=\left\langle\langle 0,1,0\rangle,\langle 1,1,1\rangle,\left\langle e_{1}, e_{2}, e_{3}\right\rangle\right\rangle$ and $M_{18}=\left\langle\langle 0,0,1\rangle,\langle 1,1,1\rangle,\left\langle f_{1}, f_{2}, f_{3}\right\rangle\right\rangle$ and $\left(r_{1} \neq 0\right.$ or $\left.r_{2} \neq 0\right)$ and $r_{1} \cdot e_{1} \cdot e_{2}+$ $\left(r_{2} \cdot e_{1} \cdot e_{3}\right)=r_{1}+r_{2} \cdot e_{2} \cdot e_{3}$ and $r_{1} \cdot f_{1} \cdot f_{2}+\left(r_{2} \cdot f_{1} \cdot f_{3}\right)=r_{1}+r_{2} \cdot f_{2} \cdot f_{3}$. Then Det $M_{9} \cdot \operatorname{Det} M_{12} \cdot \operatorname{Det} M_{16} \cdot \operatorname{Det} M_{17}=\operatorname{Det} M_{10} \cdot \operatorname{Det} M_{11} \cdot \operatorname{Det} M_{15} \cdot \operatorname{Det} M_{18}$.

In the sequel $p_{1}, p_{2}, p_{3}, p_{4}, p_{5}, p_{6}$ denote points of $\mathcal{E}_{\mathrm{T}}^{3}$.

(20) Suppose $M_{9}=\left\langle p_{1}, p_{2}, p_{5}\right\rangle$ and $M_{12}=\left\langle p_{1}, p_{3}, p_{6}\right\rangle$ and $M_{16}=\left\langle p_{2}, p_{4}, p_{6}\right\rangle$ and $M_{17}=\left\langle p_{3}, p_{4}, p_{5}\right\rangle$ and $M_{10}=\left\langle p_{1}, p_{2}, p_{6}\right\rangle$ and $M_{11}=\left\langle p_{1}, p_{3}, p_{5}\right\rangle$ and $M_{15}=\left\langle p_{2}, p_{4}, p_{5}\right\rangle$ and $M_{18}=\left\langle p_{3}, p_{4}, p_{6}\right\rangle$. Then

(i) Det $M_{9}=\left\langle\left|p_{1}, p_{2}, p_{5}\right|\right\rangle$, and

(ii) Det $M_{12}=\left\langle\left|p_{1}, p_{3}, p_{6}\right|\right\rangle$, and

(iii) $\operatorname{Det} M_{16}=\left\langle\left|p_{2}, p_{4}, p_{6}\right|\right\rangle$, and

(iv) Det $M_{17}=\left\langle\left|p_{3}, p_{4}, p_{5}\right|\right\rangle$, and

(v) Det $M_{10}=\left\langle\left|p_{1}, p_{2}, p_{6}\right|\right\rangle$, and

(vi) $\operatorname{Det} M_{11}=\left\langle\left|p_{1}, p_{3}, p_{5}\right|\right\rangle$, and

(vii) Det $M_{15}=\left\langle\left|p_{2}, p_{4}, p_{5}\right|\right\rangle$, and 
(viii) Det $M_{18}=\left\langle\left|p_{3}, p_{4}, p_{6}\right|\right\rangle$.

From now on $p_{7}, p_{8}, p_{9}$ denote points of $\mathcal{E}_{\mathrm{T}}^{3}$.

(21) Suppose $\left\langle\left|p_{1}, p_{5}, p_{9}\right|\right\rangle=0$. Then $\left\langle\left|p_{1}, p_{5}, p_{7}\right|\right\rangle \cdot\left\langle\left|p_{2}, p_{5}, p_{9}\right|\right\rangle=$ $-\left(\left\langle\left|p_{1}, p_{2}, p_{5}\right|\right\rangle \cdot\left\langle\left|p_{5}, p_{9}, p_{7}\right|\right\rangle\right)$. The theorem is a consequence of (1).

(22) Suppose $\left\langle\left|p_{1}, p_{6}, p_{8}\right|\right\rangle=0$. Then $\left\langle\left|p_{1}, p_{2}, p_{6}\right|\right\rangle \cdot\left\langle\left|p_{3}, p_{6}, p_{8}\right|\right\rangle=\left\langle\left|p_{1}, p_{3}, p_{6}\right|\right\rangle \cdot$ $\left\langle\left|p_{2}, p_{6}, p_{8}\right|\right\rangle$. The theorem is a consequence of $(1)$.

(23) Suppose $\left\langle\left|p_{2}, p_{4}, p_{9}\right|\right\rangle=0$. Then $\left\langle\left|p_{2}, p_{4}, p_{5}\right|\right\rangle \cdot\left\langle\left|p_{2}, p_{9}, p_{7}\right|\right\rangle=$ $-\left(\left\langle\left|p_{2}, p_{4}, p_{7}\right|\right\rangle \cdot\left\langle\left|p_{2}, p_{5}, p_{9}\right|\right\rangle\right)$.

(24) Suppose $\left\langle\left|p_{2}, p_{6}, p_{7}\right|\right\rangle=0$. Then $\left\langle\left|p_{2}, p_{4}, p_{7}\right|\right\rangle \cdot\left\langle\left|p_{2}, p_{6}, p_{8}\right|\right\rangle=$ $-\left(\left\langle\left|p_{2}, p_{4}, p_{6}\right|\right\rangle \cdot\left\langle\left|p_{2}, p_{8}, p_{7}\right|\right\rangle\right)$.

(25) Suppose $\left\langle\left|p_{3}, p_{4}, p_{8}\right|\right\rangle=0$. Then $\left\langle\left|p_{3}, p_{4}, p_{6}\right|\right\rangle \cdot\left\langle\left|p_{3}, p_{5}, p_{8}\right|\right\rangle=\left\langle\left|p_{3}, p_{4}, p_{5}\right|\right\rangle \cdot$ $\left\langle\left|p_{3}, p_{6}, p_{8}\right|\right\rangle$.

(26) Suppose $\left\langle\left|p_{3}, p_{5}, p_{7}\right|\right\rangle=0$. Then $\left\langle\left|p_{1}, p_{3}, p_{5}\right|\right\rangle \cdot\left\langle\left|p_{5}, p_{8}, p_{7}\right|\right\rangle=$ $-\left(\left\langle\left|p_{1}, p_{5}, p_{7}\right|\right\rangle \cdot\left\langle\left|p_{3}, p_{5}, p_{8}\right|\right\rangle\right)$. The theorem is a consequence of (1).

(27) Let us consider non zero real numbers $r_{125}, r_{136}, r_{246}, r_{345}, r_{126}, r_{135}$, $r_{245}, r_{346}, r_{157}, r_{259}, r_{597}, r_{368}, r_{268}, r_{297}, r_{247}, r_{287}, r_{358}, r_{587}$. Suppose $r_{125} \cdot r_{136} \cdot r_{246} \cdot r_{345}=r_{126} \cdot r_{135} \cdot r_{245} \cdot r_{346}$ and $r_{157} \cdot r_{259}=-\left(r_{125} \cdot r_{597}\right)$ and $r_{126} \cdot r_{368}=r_{136} \cdot r_{268}$ and $r_{245} \cdot r_{297}=-\left(r_{247} \cdot r_{259}\right)$ and $r_{247} \cdot r_{268}=$ $-\left(r_{246} \cdot r_{287}\right)$ and $r_{346} \cdot r_{358}=r_{345} \cdot r_{368}$ and $r_{135} \cdot r_{587}=-\left(r_{157} \cdot r_{358}\right)$. Then $r_{287} \cdot r_{597}=r_{297} \cdot r_{587}$.

(28) Suppose $p_{1}=\langle 1,0,0\rangle$ and $p_{2}=\langle 0,1,0\rangle$ and $p_{3}=\langle 0,0,1\rangle$ and $p_{4}=\langle 1,1$, $1\rangle$ and $p_{5}=\left\langle e_{1}, e_{2}, e_{3}\right\rangle$ and $p_{6}=\left\langle f_{1}, f_{2}, f_{3}\right\rangle$ and qfconic $\left(0,0,0, r_{1}, r_{2}\right.$, $\left.-\left(r_{1}+r_{2}\right), p_{5}\right)=0$ and qfconic $\left(0,0,0, r_{1}, r_{2},-\left(r_{1}+r_{2}\right), p_{6}\right)=0$. Then

(i) $\operatorname{qfconic}\left(0,0,0, r_{1}, r_{2},-\left(r_{1}+r_{2}\right), p_{1}\right)=0$, and

(ii) qfconic $\left(0,0,0, r_{1}, r_{2},-\left(r_{1}+r_{2}\right), p_{2}\right)=0$, and

(iii) qfconic $\left(0,0,0, r_{1}, r_{2},-\left(r_{1}+r_{2}\right), p_{3}\right)=0$, and

(iv) $\operatorname{qfconic}\left(0,0,0, r_{1}, r_{2},-\left(r_{1}+r_{2}\right), p_{4}\right)=0$, and

(v) $r_{1} \cdot e_{1} \cdot e_{2}+\left(r_{2} \cdot e_{1} \cdot e_{3}\right)=r_{1}+r_{2} \cdot e_{2} \cdot e_{3}$, and

(vi) $r_{1} \cdot f_{1} \cdot f_{2}+\left(r_{2} \cdot f_{1} \cdot f_{3}\right)=r_{1}+r_{2} \cdot f_{2} \cdot f_{3}$.

(29) Suppose $p_{1}=\langle 1,0,0\rangle$ and $p_{2}=\langle 0,1,0\rangle$ and $p_{3}=\langle 0,0,1\rangle$ and $p_{4}=\langle 1$, $1,1\rangle$ and $p_{5}=\left\langle e_{1}, e_{2}, e_{3}\right\rangle$ and $p_{6}=\left\langle f_{1}, f_{2}, f_{3}\right\rangle$ and $\left\langle\left|p_{1}, p_{2}, p_{5}\right|\right\rangle \neq 0$ and $\left\langle\left|p_{1}, p_{3}, p_{6}\right|\right\rangle \neq 0$ and $\left\langle\left|p_{2}, p_{4}, p_{6}\right|\right\rangle \neq 0$ and $\left\langle\left|p_{3}, p_{4}, p_{5}\right|\right\rangle \neq 0$ and $\left\langle\left|p_{1}, p_{2}, p_{6}\right|\right\rangle \neq 0$ and $\left\langle\left|p_{1}, p_{3}, p_{5}\right|\right\rangle \neq 0$ and $\left\langle\left|p_{2}, p_{4}, p_{5}\right|\right\rangle \neq 0$ and $\left\langle\left|p_{3}, p_{4}, p_{6}\right|\right\rangle$ $\neq 0$ and $\left\langle\left|p_{1}, p_{5}, p_{7}\right|\right\rangle \neq 0$ and $\left\langle\left|p_{2}, p_{5}, p_{9}\right|\right\rangle \neq 0$ and $\left\langle\left|p_{5}, p_{9}, p_{7}\right|\right\rangle \neq 0$ and $\left\langle\left|p_{3}, p_{6}, p_{8}\right|\right\rangle \neq 0$ and $\left\langle\left|p_{2}, p_{6}, p_{8}\right|\right\rangle \neq 0$ and $\left\langle\left|p_{2}, p_{9}, p_{7}\right|\right\rangle \neq 0$ and $\left\langle\left|p_{2}, p_{4}, p_{7}\right|\right\rangle \neq 0$ and $\left\langle\left|p_{2}, p_{8}, p_{7}\right|\right\rangle \neq 0$ and $\left\langle\left|p_{3}, p_{5}, p_{8}\right|\right\rangle \neq 0$ and $\left\langle\left|p_{5}, p_{8}, p_{7}\right|\right\rangle$ 
$\neq 0$ and $\left(r_{1} \neq 0\right.$ or $\left.r_{2} \neq 0\right)$ and $\operatorname{qfconic}\left(0,0,0, r_{1}, r_{2},-\left(r_{1}+r_{2}\right), p_{5}\right)=$ 0 and qfconic $\left(0,0,0, r_{1}, r_{2},-\left(r_{1}+r_{2}\right), p_{6}\right)=0$ and $\left\langle\left|p_{1}, p_{5}, p_{9}\right|\right\rangle=0$ and $\left\langle\left|p_{1}, p_{6}, p_{8}\right|\right\rangle=0$ and $\left\langle\left|p_{2}, p_{4}, p_{9}\right|\right\rangle=0$ and $\left\langle\left|p_{2}, p_{6}, p_{7}\right|\right\rangle=0$ and $\left\langle\left|p_{3}, p_{4}, p_{8}\right|\right\rangle$ $=0$ and $\left\langle\left|p_{3}, p_{5}, p_{7}\right|\right\rangle=0$. Then $\left\langle\left|p_{2}, p_{8}, p_{7}\right|\right\rangle \cdot\left\langle\left|p_{5}, p_{9}, p_{7}\right|\right\rangle=\left\langle\left|p_{2}, p_{9}, p_{7}\right|\right\rangle \cdot$ $\left\langle\left|p_{5}, p_{8}, p_{7}\right|\right\rangle$. The theorem is a consequence of (20), (28), (19), (21), (22), (23), (24), (25), (26), and (27).

(30) Suppose $\left\langle\left|p_{2}, p_{8}, p_{7}\right|\right\rangle \cdot\left\langle\left|p_{5}, p_{9}, p_{7}\right|\right\rangle=\left\langle\left|p_{2}, p_{9}, p_{7}\right|\right\rangle \cdot\left\langle\left|p_{5}, p_{8}, p_{7}\right|\right\rangle$. Then $\left\langle\left|p_{7}, p_{2}, p_{5}\right|\right\rangle \cdot\left\langle\left|p_{7}, p_{8}, p_{9}\right|\right\rangle=0$. The theorem is a consequence of (1).

(31) Let us consider a projective space $P_{10}$ defined in terms of collinearity, and elements $c_{1}, c_{2}, c_{3}, c_{4}, c_{5}, c_{6}, c_{7}, c_{8}, c_{9}$ of $P_{10}$. Suppose $c_{1}, c_{2}$ and $c_{4}$ are not collinear and $c_{1}, c_{2}$ and $c_{5}$ are not collinear and $c_{1}, c_{6}$ and $c_{4}$ are not collinear and $c_{1}, c_{6}$ and $c_{5}$ are not collinear and $c_{2}, c_{6}$ and $c_{4}$ are not collinear and $c_{3}, c_{4}$ and $c_{2}$ are not collinear and $c_{3}, c_{4}$ and $c_{6}$ are not collinear and $c_{3}, c_{5}$ and $c_{2}$ are not collinear and $c_{3}, c_{5}$ and $c_{6}$ are not collinear and $c_{4}, c_{5}$ and $c_{2}$ are not collinear and $c_{1}, c_{4}$ and $c_{7}$ are collinear and $c_{1}, c_{5}$ and $c_{8}$ are collinear and $c_{2}, c_{3}$ and $c_{7}$ are collinear and $c_{2}, c_{5}$ and $c_{9}$ are collinear and $c_{6}, c_{3}$ and $c_{8}$ are collinear and $c_{6}, c_{4}$ and $c_{9}$ are collinear. Then

(i) $c_{9}, c_{2}$ and $c_{4}$ are not collinear, and

(ii) $c_{1}, c_{4}$ and $c_{9}$ are not collinear, and

(iii) $c_{2}, c_{3}$ and $c_{9}$ are not collinear, and

(iv) $c_{2}, c_{4}$ and $c_{7}$ are not collinear, and

(v) $c_{2}, c_{5}$ and $c_{8}$ are not collinear, and

(vi) $c_{2}, c_{9}$ and $c_{8}$ are not collinear, and

(vii) $c_{2}, c_{9}$ and $c_{7}$ are not collinear, and

(viii) $c_{6}, c_{4}$ and $c_{8}$ are not collinear, and

(ix) $c_{6}, c_{5}$ and $c_{8}$ are not collinear, and

(x) $c_{4}, c_{9}$ and $c_{8}$ are not collinear, and

(xi) $c_{4}, c_{9}$ and $c_{7}$ are not collinear.

ProOF: For every elements $v_{102}, v_{103}, v_{100}, v_{104}$ of $P_{10}, v_{100}=v_{104}$ or $v_{104}$, $v_{100}$ and $v_{102}$ are not collinear or $v_{104}, v_{100}$ and $v_{103}$ are not collinear or $v_{102}, v_{103}$ and $v_{104}$ are collinear by [13, (5), (3)]. For every elements $v_{102}$, $v_{104}, v_{100}, v_{103}$ of $P_{10}, v_{100}=v_{103}$ or $v_{103}, v_{100}$ and $v_{102}$ are not collinear or $v_{103}, v_{100}$ and $v_{104}$ are not collinear or $v_{102}, v_{103}$ and $v_{104}$ are collinear by [13, (5), (3)]. For every elements $v_{102}, v_{103}, v_{104}, v_{101}$ of $P_{10}, v_{104}=v_{101}$ or $v_{101}, v_{104}$ and $v_{102}$ are not collinear or $v_{101}, v_{104}$ and $v_{103}$ are not collinear or $v_{102}, v_{103}$ and $v_{104}$ are collinear by [13, (2), (3)]. For every elements $v_{103}$, 
$v_{104}, v_{102}, v_{101}$ of $P_{10}, v_{102}=v_{101}$ or $v_{101}, v_{102}$ and $v_{103}$ are not collinear or $v_{101}, v_{102}$ and $v_{104}$ are not collinear or $v_{102}, v_{103}$ and $v_{104}$ are collinear by $[13,(2),(3)]$. For every elements $v_{2}, v_{101}, v_{100}$ of $P_{10}, v_{101}=v_{100}$ or $v_{100}, v_{101}$ and $v_{2}$ are not collinear or $v_{2}, v_{101}$ and $v_{100}$ are collinear by [13, $(2)]$.

In the sequel $P_{1}, P_{2}, P_{3}, P_{4}, P_{5}, P_{6}, P_{7}, P_{8}, P_{9}$ denote points of the projective space over $\mathcal{E}_{\mathrm{T}}^{3}$ and $a, b, c, d, e, f$ denote real numbers.

Let $P_{1}, P_{2}, P_{3}, P_{4}, P_{5}, P_{6}, P_{7}, P_{8}, P_{9}$ be points of the projective space over $\mathcal{E}_{\mathrm{T}}^{3}$. We say that $P_{1}, P_{2}, P_{3}, P_{4}, P_{5}, P_{6}, P_{7}, P_{8}, P_{9}$ form the Pascal configuration if and only if

(Def. 4) $P_{1}, P_{2}$ and $P_{4}$ are not collinear and $P_{1}, P_{3}$ and $P_{4}$ are not collinear and $P_{2}, P_{3}$ and $P_{4}$ are not collinear and $P_{1}, P_{2}$ and $P_{5}$ are not collinear and $P_{1}, P_{2}$ and $P_{6}$ are not collinear and $P_{1}, P_{3}$ and $P_{5}$ are not collinear and $P_{1}, P_{3}$ and $P_{6}$ are not collinear and $P_{2}, P_{4}$ and $P_{5}$ are not collinear and $P_{2}, P_{4}$ and $P_{6}$ are not collinear and $P_{3}, P_{4}$ and $P_{5}$ are not collinear and $P_{3}, P_{4}$ and $P_{6}$ are not collinear and $P_{2}, P_{3}$ and $P_{5}$ are not collinear and $P_{2}, P_{3}$ and $P_{6}$ are not collinear and $P_{4}, P_{5}$ and $P_{1}$ are not collinear and $P_{4}, P_{6}$ and $P_{1}$ are not collinear and $P_{5}, P_{6}$ and $P_{1}$ are not collinear and $P_{5}, P_{6}$ and $P_{2}$ are not collinear and $P_{1}, P_{5}$ and $P_{9}$ are collinear and $P_{1}, P_{6}$ and $P_{8}$ are collinear and $P_{2}, P_{4}$ and $P_{9}$ are collinear and $P_{2}, P_{6}$ and $P_{7}$ are collinear and $P_{3}, P_{4}$ and $P_{8}$ are collinear and $P_{3}, P_{5}$ and $P_{7}$ are collinear.

Now we state the propositions:

(32) Suppose $P_{1}, P_{2}, P_{3}, P_{4}, P_{5}, P_{6}, P_{7}, P_{8}, P_{9}$ form the Pascal configuration. Then

(i) $P_{7}, P_{2}$ and $P_{5}$ are not collinear, and

(ii) $P_{1}, P_{5}$ and $P_{7}$ are not collinear, and

(iii) $P_{2}, P_{4}$ and $P_{7}$ are not collinear, and

(iv) $P_{2}, P_{5}$ and $P_{9}$ are not collinear, and

(v) $P_{2}, P_{6}$ and $P_{8}$ are not collinear, and

(vi) $P_{2}, P_{7}$ and $P_{8}$ are not collinear, and

(vii) $P_{2}, P_{7}$ and $P_{9}$ are not collinear, and

(viii) $P_{3}, P_{5}$ and $P_{8}$ are not collinear, and

(ix) $P_{3}, P_{6}$ and $P_{8}$ are not collinear, and

(x) $P_{5}, P_{7}$ and $P_{8}$ are not collinear, and

(xi) $P_{5}, P_{7}$ and $P_{9}$ are not collinear.

The theorem is a consequence of (31). 
(33) Suppose it is not true that $a=0$ and $b=0$ and $c=0$ and $d=0$ and $e=0$ and $f=0$. Suppose that $\left\{P_{1}, P_{2}, P_{3}, P_{4}, P_{5}, P_{6}\right\} \subseteq \operatorname{conic}(a, b, c, d, e, f)$ and $P_{1}, P_{2}$ and $P_{3}$ are not collinear and $P_{1}, P_{2}$ and $P_{4}$ are not collinear and $P_{1}, P_{3}$ and $P_{4}$ are not collinear and $P_{2}, P_{3}$ and $P_{4}$ are not collinear and $P_{7}, P_{2}$ and $P_{5}$ are not collinear and $P_{1}, P_{2}$ and $P_{5}$ are not collinear and $P_{1}, P_{2}$ and $P_{6}$ are not collinear and $P_{1}, P_{3}$ and $P_{5}$ are not collinear and $P_{1}, P_{3}$ and $P_{6}$ are not collinear and $P_{1}, P_{5}$ and $P_{7}$ are not collinear and $P_{2}, P_{4}$ and $P_{5}$ are not collinear and $P_{2}, P_{4}$ and $P_{6}$ are not collinear and $P_{2}, P_{4}$ and $P_{7}$ are not collinear and $P_{2}, P_{5}$ and $P_{9}$ are not collinear and $P_{2}, P_{6}$ and $P_{8}$ are not collinear and $P_{2}, P_{7}$ and $P_{8}$ are not collinear and $P_{2}, P_{7}$ and $P_{9}$ are not collinear and $P_{3}, P_{4}$ and $P_{5}$ are not collinear and $P_{3}, P_{4}$ and $P_{6}$ are not collinear and $P_{3}, P_{5}$ and $P_{8}$ are not collinear and $P_{3}, P_{6}$ and $P_{8}$ are not collinear and $P_{5}, P_{7}$ and $P_{8}$ are not collinear and $P_{5}, P_{7}$ and $P_{9}$ are not collinear and $P_{1}, P_{5}$ and $P_{9}$ are collinear and $P_{1}, P_{6}$ and $P_{8}$ are collinear and $P_{2}, P_{4}$ and $P_{9}$ are collinear and $P_{2}, P_{6}$ and $P_{7}$ are collinear and $P_{3}, P_{4}$ and $P_{8}$ are collinear and $P_{3}, P_{5}$ and $P_{7}$ are collinear. Then $P_{7}, P_{8}$ and $P_{9}$ are collinear.

Proof: Consider $N$ being an invertible square matrix over $\mathbb{R}_{F}$ of dimension 3 such that (the homography of $N)\left(P_{1}\right)=$ Dir100 and (the homography of $N)\left(P_{2}\right)=\operatorname{Dir010}$ and (the homography of $\left.N\right)\left(P_{3}\right)=\operatorname{Dir001}$ and

(the homography of $N)\left(P_{4}\right)=$ Dir111. Consider $u_{5}$ being a point of $\mathcal{E}_{\mathrm{T}}^{3}$ such that $u_{5}$ is not zero and (the homography of $\left.N\right)\left(P_{5}\right)=$ the direction of $u_{5}$. Reconsider $p_{51}=u_{5}(1), p_{52}=u_{5}(2), p_{53}=u_{5}(3)$ as a real number. Consider $u_{6}$ being a point of $\mathcal{E}_{\mathrm{T}}^{3}$ such that $u_{6}$ is not zero and (the homography of $N)\left(P_{6}\right)=$ the direction of $u_{6}$. Reconsider $p_{61}=u_{6}(1), p_{62}=u_{6}(2)$, $p_{63}=u_{6}(3)$ as a real number. Consider $u_{7}$ being a point of $\mathcal{E}_{\mathrm{T}}^{3}$ such that $u_{7}$ is not zero and (the homography of $\left.N\right)\left(P_{7}\right)=$ the direction of $u_{7}$. Reconsider $p_{71}=u_{7}(1), p_{72}=u_{7}(2), p_{73}=u_{7}(3)$ as a real number. Consider $u_{8}$ being a point of $\mathcal{E}_{\mathrm{T}}^{3}$ such that $u_{8}$ is not zero and (the homography of $N)\left(P_{8}\right)=$ the direction of $u_{8}$. Reconsider $p_{81}=u_{8}(1), p_{82}=u_{8}(2)$, $p_{83}=u_{8}(3)$ as a real number. Consider $u_{9}$ being a point of $\mathcal{E}_{\mathrm{T}}^{3}$ such that $u_{9}$ is not zero and (the homography of $\left.N\right)\left(P_{9}\right)=$ the direction of $u_{9}$. Reconsider $p_{91}=u_{9}(1), p_{92}=u_{9}(2), p_{93}=u_{9}(3)$ as a real number. Consider $a_{2}, b_{2}, c_{2}, d_{2}, e_{2}, f_{2}$ being real numbers such that it is not true that $a_{2}=0$ and $b_{2}=0$ and $c_{2}=0$ and $d_{2}=0$ and $e_{2}=0$ and $f_{2}=0$. (the homography of $N)\left(P_{1}\right) \in \operatorname{conic}\left(a_{2}, b_{2}, c_{2}, d_{2}, e_{2}, f_{2}\right)$ and (the homography of $\left.N\right)\left(P_{2}\right) \in$ $\operatorname{conic}\left(a_{2}, b_{2}, c_{2}, d_{2}, e_{2}, f_{2}\right)$ and (the homography of $\left.N\right)\left(P_{3}\right) \in \operatorname{conic}\left(a_{2}, b_{2}, c_{2}\right.$, $\left.d_{2}, e_{2}, f_{2}\right)$ and (the homography of $\left.N\right)\left(P_{4}\right) \in \operatorname{conic}\left(a_{2}, b_{2}, c_{2}, d_{2}, e_{2}, f_{2}\right)$ and (the homography of $N)\left(P_{5}\right) \in \operatorname{conic}\left(a_{2}, b_{2}, c_{2}, d_{2}, e_{2}, f_{2}\right)$ and (the homography of $N)\left(P_{6}\right) \in \operatorname{conic}\left(a_{2}, b_{2}, c_{2}, d_{2}, e_{2}, f_{2}\right)$. Consider $P$ being a point of the pro- 
jective space over $\mathcal{E}_{\mathrm{T}}^{3}$ such that the direction of $[1,0,0]=P$ and for every element $u$ of $\mathcal{E}_{\mathrm{T}}^{3}$ such that $u$ is not zero and $P=$ the direction of $u$ holds $\operatorname{qfconic}\left(a_{2}, b_{2}, c_{2}, d_{2}, e_{2}, f_{2}, u\right)=0 . \operatorname{qfconic}\left(a_{2}, b_{2}, c_{2}, d_{2}, e_{2}, f_{2},[1,0,0]\right)=0$ and qfconic $\left(a_{2}, b_{2}, c_{2}, d_{2}, e_{2}, f_{2},[0,1,0]\right)=0$ and qfconic $\left(a_{2}, b_{2}, c_{2}, d_{2}, e_{2}, f_{2}\right.$, $[0,0,1])=0$ and $\operatorname{qfconic}\left(a_{2}, b_{2}, c_{2}, d_{2}, e_{2}, f_{2},[1,1,1]\right)=0$ and qfconic $\left(a_{2}, b_{2}\right.$, $\left.c_{2}, d_{2}, e_{2}, f_{2},\left[p_{51}, p_{52}, p_{53}\right]\right)=0$ and $\operatorname{qfconic}\left(a_{2}, b_{2}, c_{2}, d_{2}, e_{2}, f_{2},\left[p_{61}, p_{62}, p_{63}\right]\right)$ $=0$ by [4, (10)], [8, (3)]. Reconsider $a_{7}=a_{2}, b_{7}=b_{2}, c_{10}=c_{2}, d_{3}=d_{2}$, $e_{4}=e_{2}, f_{4}=f_{2}$ as an element of $\mathbb{R}_{\mathrm{F}} \cdot a_{7}=0$ and $b_{7}=0$ and $c_{10}=$ 0. $a_{7}=0$ and $b_{7}=0$ and $c_{10}=0$ and $d_{3}+e_{4}+f_{4}=0$. Reconsider $p_{2}=\langle 0,1,0\rangle, p_{5}=\left\langle p_{51}, p_{52}, p_{53}\right\rangle, p_{7}=\left\langle p_{71}, p_{72}, p_{73}\right\rangle, p_{8}=\left\langle p_{81}, p_{82}, p_{83}\right\rangle$, $p_{9}=\left\langle p_{91}, p_{92}, p_{93}\right\rangle$ as a point of $\mathcal{E}_{\mathrm{T}}^{3} .\left\langle\left|p_{7}, p_{2}, p_{5}\right|\right\rangle \neq 0$ by [3, (102)], [8, (3)], [3, (43)], [4, (10)]. $\left\langle\left|p_{2}, p_{8}, p_{7}\right|\right\rangle \cdot\left\langle\left|p_{5}, p_{9}, p_{7}\right|\right\rangle=\left\langle\left|p_{2}, p_{9}, p_{7}\right|\right\rangle \cdot\left\langle\left|p_{5}, p_{8}, p_{7}\right|\right\rangle$. $\left\langle\left|p_{7}, p_{2}, p_{5}\right|\right\rangle \cdot\left\langle\left|p_{7}, p_{8}, p_{9}\right|\right\rangle=0$.

(34) Suppose it is not true that $a=0$ and $b=0$ and $c=0$ and $d=0$ and $e=0$ and $f=0$. Suppose that $\left\{P_{1}, P_{2}, P_{3}, P_{4}, P_{5}, P_{6}\right\} \subseteq \operatorname{conic}(a, b, c, d, e, f)$ and $P_{1}, P_{2}$ and $P_{3}$ are not collinear and $P_{1}, P_{2}, P_{3}, P_{4}, P_{5}, P_{6}, P_{7}, P_{8}, P_{9}$ form the Pascal configuration. Then $P_{7}, P_{8}$ and $P_{9}$ are collinear. The theorem is a consequence of (32) and (33).

Note that $\mathcal{E}_{\mathrm{T}}^{3}$ is up 3 -dimensional.

(35) Suppose it is not true that $a=0$ and $b=0$ and $c=0$ and $d=0$ and $e=0$ and $f=0$. Suppose that $\left\{P_{1}, P_{2}, P_{3}, P_{4}, P_{5}, P_{6}\right\} \subseteq \operatorname{conic}(a, b, c, d, e, f)$ and $P_{1}, P_{2}$ and $P_{3}$ are collinear and $P_{1}, P_{2}, P_{3}, P_{4}, P_{5}, P_{6}, P_{7}, P_{8}, P_{9}$ form the Pascal configuration. Then $P_{7}, P_{8}$ and $P_{9}$ are collinear.

Proof: Consider $N$ being an invertible square matrix over $\mathbb{R}_{F}$ of dimension 3 such that (the homography of $N)\left(P_{1}\right)=$ Dir100 and (the homography of $N)\left(P_{2}\right)=\operatorname{Dir010}$ and (the homography of $\left.N\right)\left(P_{4}\right)=$ Dir001 and (the homography of $N)\left(P_{5}\right)=$ Dir111. Consider $u_{3}$ being a point of $\mathcal{E}_{\mathrm{T}}^{3}$ such that $u_{3}$ is not zero and (the homography of $\left.N\right)\left(P_{3}\right)=$ the direction of $u_{3}$. Reconsider $p_{31}=u_{3}(1), p_{32}=u_{3}(2), p_{33}=u_{3}(3)$ as a real number. Consider $u_{6}$ being a point of $\mathcal{E}_{\mathrm{T}}^{3}$ such that $u_{6}$ is not zero and (the homography of $N)\left(P_{6}\right)=$ the direction of $u_{6}$. Reconsider $p_{61}=u_{6}(1), p_{62}=u_{6}(2)$, $p_{63}=u_{6}(3)$ as a real number. Consider $a_{2}, b_{2}, c_{2}, d_{2}, e_{2}, f_{2}$ being real numbers such that it is not true that $a_{2}=0$ and $b_{2}=0$ and $c_{2}=0$ and $d_{2}=0$ and $e_{2}=0$ and $f_{2}=0$ and (the homography of $\left.N\right)\left(P_{1}\right) \in$ $\operatorname{conic}\left(a_{2}, b_{2}, c_{2}, d_{2}, e_{2}, f_{2}\right)$ and (the homography of $\left.N\right)\left(P_{2}\right) \in \operatorname{conic}\left(a_{2}, b_{2}, c_{2}\right.$, $\left.d_{2}, e_{2}, f_{2}\right)$ and (the homography of $\left.N\right)\left(P_{3}\right) \in \operatorname{conic}\left(a_{2}, b_{2}, c_{2}, d_{2}, e_{2}, f_{2}\right)$ and (the homography of $N)\left(P_{4}\right) \in \operatorname{conic}\left(a_{2}, b_{2}, c_{2}, d_{2}, e_{2}, f_{2}\right)$ and (the homography of $N)\left(P_{5}\right) \in \operatorname{conic}\left(a_{2}, b_{2}, c_{2}, d_{2}, e_{2}, f_{2}\right)$ and (the homography of $\left.N\right)\left(P_{6}\right) \in$ conic $\left(a_{2}, b_{2}, c_{2}, d_{2}, e_{2}, f_{2}\right)$. Consider $P$ being a point of the projective space over $\mathcal{E}_{\mathrm{T}}^{3}$ such that the direction of $[1,0,0]=P$ and for every ele- 
ment $u$ of $\mathcal{E}_{\mathrm{T}}^{3}$ such that $u$ is not zero and $P=$ the direction of $u$ holds $\operatorname{qfconic}\left(a_{2}, b_{2}, c_{2}, d_{2}, e_{2}, f_{2}, u\right)=0 . \operatorname{qfconic}\left(a_{2}, b_{2}, c_{2}, d_{2}, e_{2}, f_{2},[1,0,0]\right)=0$ and qfconic $\left(a_{2}, b_{2}, c_{2}, d_{2}, e_{2}, f_{2},[0,1,0]\right)=0$ and qfconic $\left(a_{2}, b_{2}, c_{2}, d_{2}, e_{2}, f_{2}\right.$, $[0,0,1])=0$ and $\operatorname{qfconic}\left(a_{2}, b_{2}, c_{2}, d_{2}, e_{2}, f_{2},[1,1,1]\right)=0$ and qfconic $\left(a_{2}, b_{2}\right.$, $\left.c_{2}, d_{2}, e_{2}, f_{2},\left[p_{31}, p_{32}, p_{33}\right]\right)=0$ and $\operatorname{qfconic}\left(a_{2}, b_{2}, c_{2}, d_{2}, e_{2}, f_{2},\left[p_{61}, p_{62}, p_{63}\right]\right)$ $=0$ by [4, (10)], [8, (3)]. Reconsider $a_{7}=a_{2}, b_{7}=b_{2}, c_{10}=c_{2}, d_{3}=d_{2}$, $e_{4}=e_{2}, f_{4}=f_{2}$ as an element of $\mathbb{R}_{F} \cdot a_{7}=0$ and $b_{7}=0$ and $c_{10}=0$. $a_{7}=0$ and $b_{7}=0$ and $c_{10}=0$ and $d_{3}+e_{4}+f_{4}=0$. Reconsider $p_{1}=\langle 1$, $0,0\rangle, p_{2}=\langle 0,1,0\rangle, p_{3}=\left\langle p_{31}, p_{32}, p_{33}\right\rangle$ as a point of $\mathcal{E}_{\mathrm{T}}^{3} \cdot\left\langle\left|p_{1}, p_{2}, p_{3}\right|\right\rangle=0$ by [3, (102)], [10, (23)], [9, (25)], [4, (10)]. $p_{31} \neq 0$ and $p_{32} \neq 0$ by [8, (2), (8), (4)].

\section{(36) PASCAL's THEOREM:}

Suppose it is not true that $a=0$ and $b=0$ and $c=0$ and $d=0$ and $e=0$ and $f=0$. Suppose that $\left\{P_{1}, P_{2}, P_{3}, P_{4}, P_{5}, P_{6}\right\} \subseteq \operatorname{conic}(a, b, c, d, e, f)$ and $P_{1}, P_{2}, P_{3}, P_{4}, P_{5}, P_{6}, P_{7}, P_{8}, P_{9}$ form the Pascal configuration. Then $P_{7}$, $P_{8}$ and $P_{9}$ are collinear. The theorem is a consequence of (35) and (34).

\section{REFERENCES}

[1] Jesse Alama. Escape to Mizar for ATPs. arXiv preprint arXiv:1204.6615, 2012.

[2] Grzegorz Bancerek, Czesław Byliński, Adam Grabowski, Artur Korniłowicz, Roman Matuszewski, Adam Naumowicz, Karol Pąk, and Josef Urban. Mizar: State-of-the-art and beyond In Manfred Kerber, Jacques Carette, Cezary Kaliszyk, Florian Rabe, and Volker Sorge, editors, Intelligent Computer Mathematics, volume 9150 of Lecture Notes in Computer Science, pages 261-279. Springer International Publishing, 2015. ISBN 978-3319-20614-1. doi 10.1007/978-3-319-20615-8_17.

[3] Roland Coghetto. Homography in $\mathbb{R P}^{2}$. Formalized Mathematics, 24(4):239-251, 2016. doi:10.1515/forma-2016-0020.

[4] Roland Coghetto. Group of homography in real projective plane. Formalized Mathematics, 25(1):55-62, 2017. doi $10.1515 /$ forma-2017-0005

[5] Agata Darmochwal. The Euclidean space Formalized Mathematics, 2(4):599-603, 1991.

[6] Adam Grabowski. Solving two problems in general topology via types. In Types for Proofs and Programs, International Workshop, TY PES 2004, Jouy-en-Josas, France, December 15-18, 2004, Revised Selected Papers, pages 138-153, 2004. doi 10.1007/11617990_9.

[7] Adam Grabowski. Mechanizing complemented lattices within Mizar system. Journal of Automated Reasoning, 55:211-221, 2015. doi:10.1007/s10817-015-9333-5.

[8] Kanchun, Hiroshi Yamazaki, and Yatsuka Nakamura. Cross products and tripple vector products in 3-dimensional Euclidean space. Formalized Mathematıcs, 11(4):381-383, 2003.

[9] Woiciech Leończuk and Krzysztof Prażmowski. A construction of analytical projective space. Formalized Mathematics, 1(4):761-766, 1990.

[10] Wojciech Leończuk and Krzysztof Prażmowski. Projective spaces - part I Formalized Mathematics, 1(4):767-776, 1990.

[11] Jürgen Richter-Gebert. Pappos's Theorem: Nine Proofs and Three Variations, pages 3-31. Springer Berlin Heidelberg, 2011. ISBN 978-3-642-17286-1. doi 10.1007/978-3-642-172861_1.

[12] Piotr Rudnicki and Josef Urban. Escape to ATP for Mizar. In First International Workshop on Proof eXchange for Theorem Proving-PxTP 2011, 2011.

[13] Wojciech Skaba. The collinearity structure. Formalized Mathematics, 1(4):657-659, 1990. 
[14] Nobuyuki Tamura and Yatsuka Nakamura. Determinant and inverse of matrices of real elements. Formalized Mathematics, 15(3):127-136, 2007. doi 10.2478/v10037-007-0014-7.

Received June 27, 2017

The English version of this volume of Formalized Mathematics was financed under agreement 548/P-DUN/2016 with the funds from the Polish Minister of Science and Higher Education for the dissemination of science. 